Z. Klin. Chem. Klin. Biochem.

12. Jg. 1974, S. $193-197$

\title{
Spezifische Bestimmung von Corticosteron und Cortisol im Nanogramm-Bereich
}

\author{
Von C. Hesse, K. Pietrzik und D. Hötzel \\ Aus dem Institut für Ernährungswissenschaft (Direktor: Prof. Dr. D. Hötzel) der Universität Bonn
}

(Eingegangen am 24. September 1973/22. Februar 1974)

\begin{abstract}
Die spezifische chemische Bestimmung von Corticosteron und Cortisol mit der schnellen Flüssigkeitschromatographie wird beschrieben. Von der Erfassungsgrenze von $5 \mathrm{ng}$ (Corticosteron) bzw. $10 \mathrm{ng}$ (Cortisol) bis zu wenigstens $1 \mu \mathrm{g}$ verläuft die Kalibrierungskurve linear. Die scheinbare Wiederfindung beträgt für Corticosteron $102 \pm 6 \%$, für Cortisol $98 \pm 4 \%$. Die Richtigkeit der Methode wurde durch Vergleichsanalysen nach einer Referenzmethode und nach der Proteinbindungsmethodc bclegt, während sich die Spezifität durch Auftrennung einer synthetischen Mischung, durch die Untersuchung von Plasmaproben adrenalektomierter Ratten und durch Aufstockversuche mit Cortisol zu Rattenplasma demonstrieren ließ. Das Verfahren hat sich bei über 1500 Corticosteronbestimmungen als spezifische Routinemethode bewährt.
\end{abstract}

\section{Specific determination of corticosterone and cortisol in the nanogramm-range}

The specific chemical determination of corticosterone and cortisol was performed by high-speed liquid chromatography. The calibration was linear from the detection limit of $5 \mathrm{ng}$ for corticosterone and $10 \mathrm{ng}$ for cortisol up to at least $1 \mu \mathrm{g}$ for each hormone. The apparent recovery was $102 \pm 6 \%$ for corticosterone, and $98 \pm 4 \%$ for cortisol. The accuracy of the method was determined by comparison with a reference method, and with a protein binding assay. The precision was found to be within $\pm 6 \%$. The specifity of the analysis was tested by the separation of a synthetic mixture, by analysis of plasma from adrenalectomized rats and by the addition of known amounts of cortisol to samples of rat plasma. The method was used successfully for the assay of at least 1500 samples of rat plasma for corticosterone.

Bei Pantothensäuremangel sind eine erhöhte Insulinempfindlichkeit und das Ausbleiben des Eosinophilensturzes nach Gabe von adrenocorticotropem Hormon (ACTH) beobachtet worden (1). Beiden Befunden könnte eine verminderte Produktionsfähigkeit der Nebennierenrinde für die Glucocorticoide zugrunde liegen. Es erschien daher interessant, Zusammenhänge zwischen dem Versorgungszustand an Pantothensäure und der Produktionsfähigkeit der Nebennierenrinde für Steroidhormone nachzuweisen.

Da die Ratte als Glucocorticoid überwiegend Corticosteron, der Mensch aber Cortisol produziert, sollte eine spezifische chemische Bestimmungsmethode entwickelt werden, die beim Einsatz kleiner Probenmengen den Nachweis der natürlichen Hormone und ihrer synthetischen Analogen nebeneinander gestattet.

Prinzipiell können solche Analysen unter anderem mit der Gaschromatographie oder der schnellen Flüssigkeitschromatographie durchgefuhrt werden. Bei der Flüssigchromatographie entfallen die Derivatisierungsschritte, so daß sich hier die Probenvorbereitung gegenüber der Gaschromatographie erheblich vereinfachen und beschleunigen läßt. Aufgrund ihrer Carbonylfunktion in Konjugation mit einer Doppelbindung können die Corticosteroide mit Spektralphotometerdetektoren bei $240 \mathrm{~nm}$ sehr empfindlich und bequem nachgewiesen werden $\left(\epsilon \sim 10^{4} \mathrm{~cm}^{2} / \mathrm{mol}\right)$. Trennleistungen und Analysenzeiten wie bei der Gaschromatographie sind durchaus erreichbar. Da die bisher publizierten Verfahren nicht die Analyse der natürlichen Hormone und der häufigsten Analogen nebeneinander gestatteten, haben wir eine eigene Methode entwickelt, über die hier berichtet werden soll(2-5).

\section{Material und Methoden}

Dichlormethan (Baker, Groß-Gerau)

wurde vor Gebrauch sorgfältig destilliert; eine weitergehende Reinigung war nicht erforderlich.

Äthanol

der Uvasol-Qualität (Merck, Darmstadt) wurde vor der Verwendung.über $\mathrm{Na}-\mathrm{Pb}$-Legierung (Dri-Na, Baker) destilliert.

Steroide

waren von der Firma Serva bezogen und ohne weitere Reinigung verwendbar. Die Stoffe waren in der Flüssigkeitschromatographie und in der zweidimensionalen Dünnschichtchromatographie (I: $\mathrm{CH}_{2} \mathrm{Cl}_{2}$ mit 2\% Äthanol; II: Benzol/Aceton/Wasser $50 \mathrm{ml}+$ $50 \mathrm{ml}+1 \mathrm{ml}$ ) einheitlich. Felddesorptions-Massenspektren von Cortisol und Prednisolon zeigten nur den Peak des Molekülions und des doppelt geladenen Molekülions.

Die Trennungen führten wir mit einem Flüssigchromatographen S 200 P mit Photometerdetektor PM 4 CHR durch (Siemens, Karlsruhe und Zeiß, Obcrkochen). Bei einer spektralen Bandbreite von $\pm 10 \mathrm{~nm}$ betrug die Meßwellenlänge $240 \mathrm{~nm}$. Die Säule (Tantal) hatte eine Länge von $30 \mathrm{~cm}$ und einen inneren Durchmesser von $2 \mathrm{~mm}$. Sie war mit Spherosil XOA 400 des Korngrößenbereichs 4-8 $\mu \mathrm{m}$ gefüllt (Pechiney-Saint-Gobain). Für den Erfolg einer Trennung ist die Verwendung einer Säule, die mit engklassiertem Trägermaterial gefüllt ist, entscheidend. 
Als mobile Phase dientc der unpolare Anteil einer Mischung von Dichlormethan, Äthanol und Wasser $(94,8 \mathrm{ml}+3,5 \mathrm{ml}+1,7 \mathrm{ml})$. Dieses Dreikomponenten-Zweiphasensystem ist an anderer Stelle ausführlich beschrieben worden (6). Die mobile Phase wird wie folgt gewonnen: In einem 2-1-Meßkolben wurden $34 \mathrm{ml}$ Wasser, $70 \mathrm{ml}$ Äthanol und $1896 \mathrm{ml}$ Dichlormethan bei $25^{\circ}$ über Nacht intensiv gerührt. Nach der Phasentrennung wurde die polare Oberphase (etwa $40 \mathrm{ml}$ ) verworfen und die Unterphase durch ein Faltenfilter in das Gerät gegeben. Beim ersten Einsatz einer solchen Phase muß die Säule zunächst durch Durchpumpen des Fließmittels während einiger Stunden konditioniert werden. Unwirksame Säulen können auf einfache Weise wieder brauchbär gemacht werden, indem für etwa eine Stunde Dioxan hindurchgepumpt wird. Eine derartig behandelte Säule befindet sich seit nunmehr über zwei Jahren im Betrieb.

Die Spezifitätsprüfung wurde in Anlehnung an die von Pirke und Stamm publizierte Methode durchgefürt (7). Zur Endbestimmung wurde statt der fluorimetrischen die flüssigkeitschromatographische Methode benutzt.

Die Auswertung aller quantitativen Analysen erfolgte nach der Methode des inneren Standards. Aufarbeitungsverluste und Dosierfehler lassen sich so auf einfache und sichere Art unschädlich machen. Dabei wurde aus technischen Gründen für die Bestimmung von Cortisol Prednison, für die Bestimmung von Corticosteron Prednisolon als Standardsubstanz verwendet.

\section{Extraktion und Analyse}

$\mathrm{Zu} 1,00 \mathrm{ml}$ Humanserum in einem $50 \mathrm{ml}$-Erlenmeyerkolben gibt man $0,50 \mathrm{ml}$ einer Lösung von $2 \mathrm{mg} / 1$ Prednison, $0,1 \mathrm{ml} 2 \mathrm{~mol} / 1$ $\mathrm{NaOH}$ und $10 \mathrm{ml}$ Dichlormethan. Es hat sich gezeigt, daß aus dem alkalischen Milieu heraus die Extrakte sauberer werden, ohne daß eine Ausbeuteverminderung eintritt.

Man rührt $15 \mathrm{~min}$ auf einem Magnetrührer und trennt die organische Phase in einem Scheidetrichter ab. Die wäßrige Phase wird in gleicher Weise noch zweimal mit je $10 \mathrm{ml}$ Dichlormethan extrahiert. Die vereinigten Extrakte werden zur Trockene gebracht. Man spült den Rückstand mehrfach in kleinere Gefäße über, dabei wird zwischendurch immer zur Trockene eingedampft. Schließlich wird mit $40 \mu$ Dichlormethan aufgefüllt und die Probe in die Dosiervorrichtung des Chromatographen gegeben. Bei einem Durchfluß von $1 \mathrm{ml} / \mathrm{min}$ wurde mit $1 / 32$ Extinktionseinheit für Schreibervollausschlag gearbeitet. Der Rauschpegel lag in diesem Fall bei weniger als $1 \%$ des Vollausschlags.

Die Peaks der Probe und des inneren Standards identifiziert man anhand von Vergleichschromatogrammen reiner Lösungen. Die weitere Auswertung wird so durchgefuhrt, wie sie bei Kaiser beschrieben ist (8).

Für die Analyse von Rattenplasma werden $0,5 \mathrm{ml}$ Plasma, $1,0 \mathrm{ml}$ Prednisolonlösung $(2 \mathrm{mg} / \mathrm{l})$ und $0,1 \mathrm{ml} 2 \mathrm{~mol} / 1 \mathrm{NaOH}$ eingesetzt.

\section{Ergebnisse und Diskussion}

\section{Aufbau der Bestimmung}

Prinzipiell ist es möglich, die gleiche Trennung mit einem selektiven Trennsystem und einer Säule geringerer Effizienz oder aber mit einer hochwirksamen Säule und einem weniger selektiven chromatographischen Milieu zu erreichen. Huber konnte allerdings zeigen, daß die Länge einer Säule und ihre Trennstufenhöhe Einfluß auf die Verdünnung einer Probe während ihrer Wanderung durch die Säule und damit auf die kleinste Menge haben, in der sie noch nachgewiesen werden kann (9). Es ist bei Spurenanalysen daher grundsätzlich notwendig, hochwirksame Säulen einzusetzen. Bei Verwendung feiner, enggesiebter Teilchen und von Suspensionsmethoden bei der Füllung ist es möglich, Trennleistungen von 10000 Böden pro Meter zu erreichen.

In Abbildung 1 ist die Trennung einer Mischung auf einer solchen Säule dargestellt.



Abb. 1. Trennung von Progesteron, Testosteron, Corticosteron, Prednison, Cortison, Aldosteron, Cortisol, Prednisolon. Analysenzeit: $10 \mathrm{~min}$, Durchfluß: $1 \mathrm{ml} / \mathrm{min}$, Detektorwellenlänge: $240 \mathrm{~nm}$.

In den Abbildungen 2 und 3 sind Chromatogramme von Extrakten von Human- bzw. Rattenseren gezeigt. In beiden Fällen wurde der innere Standard vor der Extraktion zugesetzt, im Falle der Abbildung 3 außerdem noch eine Spur Aldosteron, um die Position dieses Steroids im Chromatogramm zu markieren.

Zur Bestimmung der Linearität haben wir eine konstante Menge des inneren Standards mit steigenden Mengen des Hormons zusammen in Dichlormethan gelöst, analysiert

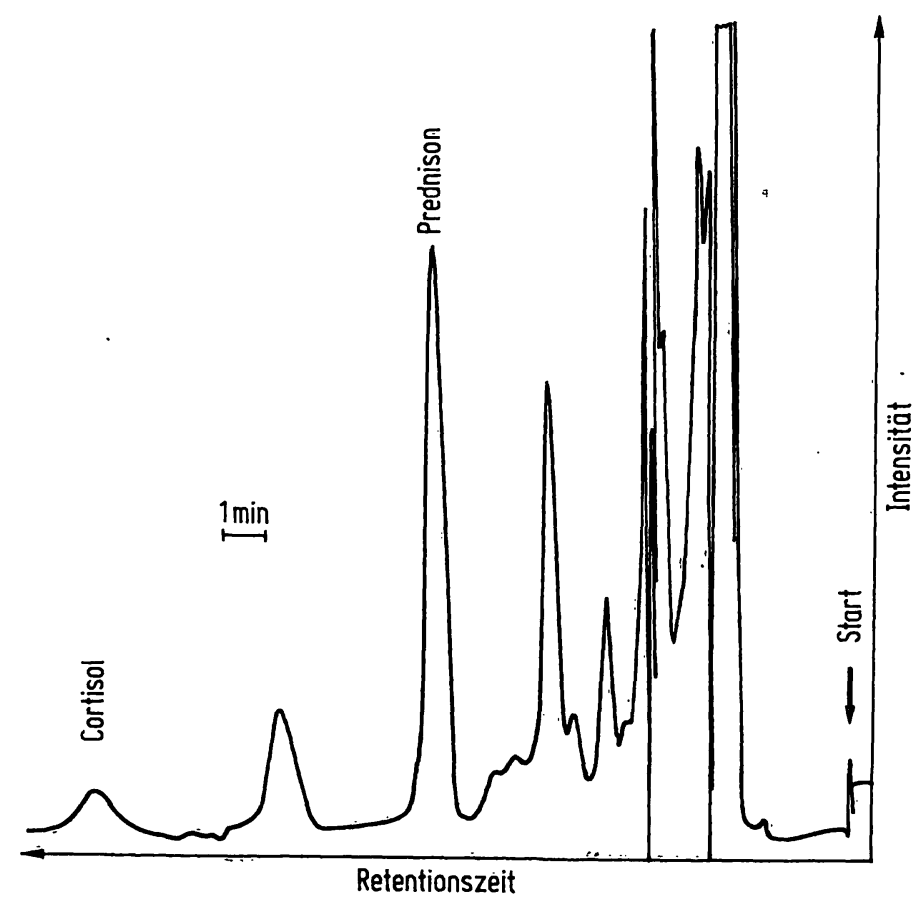

Abb. 2. Chromatogramm eines Extraktes aus Humanserum. Bedingungen wie bei Abb. 1. 


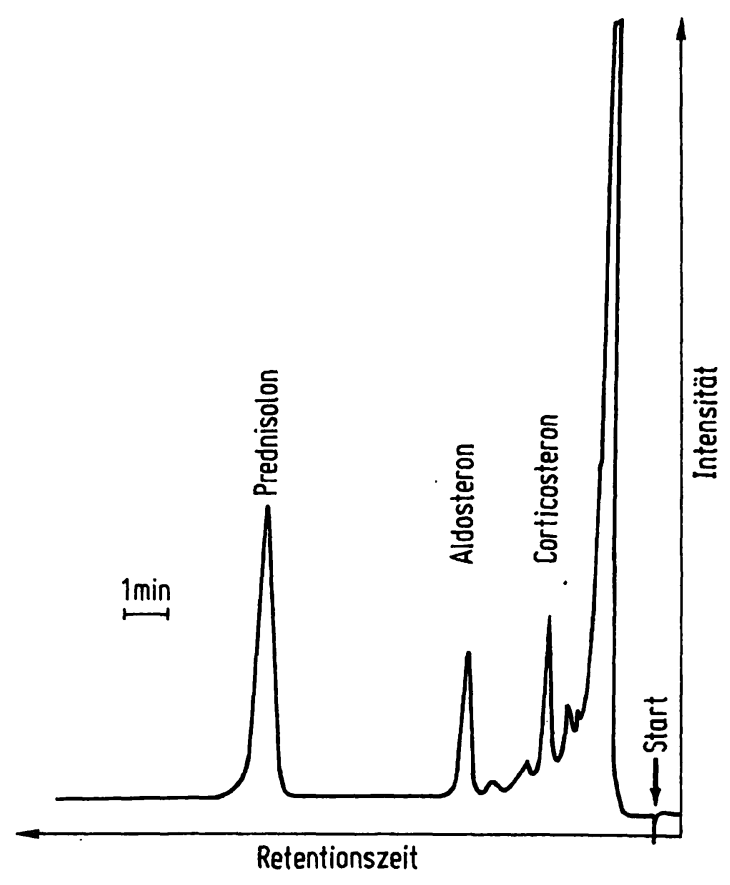

Abb. 3. Chromatogramm eines Extraktes aus Rattenplasma. Bedingungen wie Abb. 1.

und die Quotienten aus den Peakflächen des Hormons und des inneren Standards gegen die eingesetzte Menge des Hormons aufgetragen. Erwartungsgemäß geht die Eichgerade durch den Nullpunkt des Koordinatensystems (Abb. 4). Die dreimalige Extraktion der Proben wurde durchgeführt, um auch bei sehr verschiedenen Verteilungskoeffizienten der Analysensubstanzen und des inneren Standards die gleiche Extraktionsausbeute zu erhalten. Bei einem Verteilungsverhältnis von $70 \%$ zugunsten der lipophilen Phase erhält man nach dreimaliger Extraktion eine Ausbeute von 97\%, bei einem entsprechenden Verhältnis von $90 \%$ eine solche von $99,9 \%$. Diese Unterschiede liegen im Rahmen der derzeit erreichten Fehlergrenze. Erwartungsgemäß unterschieden sich die Normie-

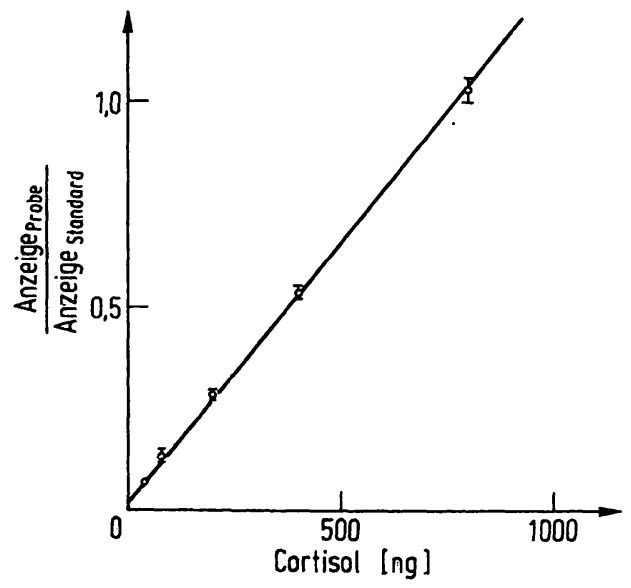

Abb. 4. Linearität der Anzeige für Cortisol. Regressionsgleichung: $y=0,79 x-0,01 ; r=0,9.99$. Für Corticosteron wird eine entsprechende Eichgerade erhalten. Regressionsgleichung: $y=1,29 x-0,02 ; x=0,999$. rungsfaktoren aus organischer Lösung und aus der Extraktion nicht. Sie betrugen für Cortisol 1,37 $\pm 0,12$ ohne und 1,25 $\pm 0,15$ mit Extraktion. Es handelt sich dabei um Mittelwerte über ein halbes Jahr und jeweils über 20 Messungen. Für Corticosteron lauten die Werte 1,25 \pm 0,1 und 1,19 $\pm 0,11$. Besonders hervorzuheben ist der große lineare Bereich der Bestimmungsmethode. Mehrfachanalysen mit geändertem Probeneinsatz lassen sich dadurch meist vermeiden. In Tabelle 1 sind einige für Cortisol erhaltene Ergebnisse dargestellt. Für Corticosteron erhielten wir zwischen $100 \mathrm{ng}$ und $1000 \mathrm{ng}$ scheinbare Wiederfindungsraten von $102 \pm 6 \%$, für Cortisol $98 \pm 4 \%$.

Tab. 1. Wiederfindungsversuche mit Cortisol

\begin{tabular}{ccc}
\hline $\begin{array}{c}\text { Eingewogen } \\
\text { [ng] }\end{array}$ & $\begin{array}{c}\text { Gefunden } \\
\text { [ng] }\end{array}$ & $\begin{array}{c}\text { Wiederfindung } \\
{[\%]}\end{array}$ \\
\hline 100 & 105 & 105 \\
200 & 186 & 93 \\
200 & 201 & 100 \\
500 & 467 & 93 \\
500 & 483 & 97 \\
700 & 688 & 98 \\
700 & 698 & 100 \\
1000 & 1006 & 100 \\
1000 & 1001 & 100 \\
& & $\overline{\mathrm{x}} \pm \mathrm{s}=98 \pm 4$ \\
\hline
\end{tabular}

Die Übertragung der Methode auf biologisches Material ließ sich nunmehr ohne Schwierigkeiten durchfüren. Da der innere Standard der Serumprobe vor der Extraktion zugesetzt wird, kompensieren sich die Verluste von Probe und Standard gegenseitig, so daß normalerweise auf einen radioaktiven endogenen Standard verzichtet werden kann. Eine Person kann pro Arbeitstag bis zu 30 Analysen bearbeiten, wenn fünf Rotationsverdampfer zum Eindampfen des Lösungsmittels zur Verfügung stehen. Der Zeitbedarf vom Beginn der Analyse bis zum Vorliegen der ersten Resultate beträgt etwa drei Stunden. Bisher haben wir über 1500 Corticosteronbestimmungen aus Rat̂tenplasma auf diese Weise durchführen können. Einen Überblick über die dort erhaltenen Ergebnisse gibt die Tabelle 2. Die Resultate aus einem Vitaminmangelversuch werden an anderer Stelle veröffentlicht (10).

\section{Zuverlässigkeitskriterien}

\section{Spezifität}

Aufgrund der Detektionsmethode werden Verbindungen, die bei $240 \mathrm{~nm}$ keine Strahlung absorbieren, nicht angezeigt. Unter den oben genannten Bedingungen lassen

Tab. 2. Corticosteronspiegel in Rattenplasma

\begin{tabular}{ll}
\hline Corticosteron $[\mu \mathrm{g} / \mathrm{l}]$ & Vorbchandlung \\
\hline $265 \pm 60$ & Basalwert, ohne Vorbehandlung $(\mathrm{n}=6)$ \\
$937 \pm 400$ & Vorbehandlung mit $0,1 \mathrm{mg} \mathrm{ACTH} / \mathrm{je}$ \\
76 & Ratte $(\mathrm{n}=54)$ \\
65 & Dic Synthese des Corticosterons wurde \\
& $\begin{array}{l}\text { durch i.m. Injektion von Cortisol-Sus- } \\
\text { pension unterdrückt. }\end{array}$ \\
\hline
\end{tabular}


sich folgende Hormone und Hormonanalogen voneinander trennen: Progesteron, Testosteron, Corticosteron, Frednison, Cortison, Aldosteron, Fluocortolọn, Dexamethason, Cortisol und Prednisolon. Cholesterin, das nur mit dem Differentialrefraktometer angezeigt werden kann, läuft mit der Lösungsmittelfront, ebenso wie Desoxycorticosteron und unpolar veresterte Derivate (Benzoate, Acetate). Höher hydroxylierte Derivate würden nach dem Prednisolon durchbrechen. Hier werden denn auch gelegentlich Banden beobachtet. Chromatogramme von Extrakten aus Rattenplasma zeigen an der Stelle des Cortisols nur die Grundlinie. Untersucht man Proben von frisch adrenalektomierten Ratten, läßt sich an der Stelle des Corticosterons kein anderer Stoff nachweisen. Das bedeutet, daß eine Interferenz durch andere Extraktstoffe nicht eintritt. Aufstockversuche mit Cortisol zu Rattenplasma gaben befriedigende Resultate (Tab. 3).

Tab. 3. Aufstockversuch mit Cortisol zu Rattenplasma

\begin{tabular}{ccc}
\hline $\begin{array}{c}\text { Eingesetzt } \\
\text { [ng] }\end{array}$ & $\begin{array}{c}\text { Gefunden } \\
\text { [ng] }\end{array}$ & $\begin{array}{c}\text { Wiederfindung } \\
{[\%]}\end{array}$ \\
\hline 0 & 0 & - \\
50 & 55 & 110 \\
100 & 98 & 98 \\
200 & 224 & 112 \\
\hline
\end{tabular}

\section{Richtigkeit}

Zur Überprüfung von Spezifität und Richtigkeit wurden Plasmaproben mit einem Cortisolgehalt von etwa $40-300 \mu \mathrm{g} / 1$ nach einfacher Extraktion und nach dünnschichtchromatographischer Auftrennung (7) untersucht und die Ergebnisse in der von Pirke und Stamm angegebenen Art zur Berechnung des Ähnlichkeitskoeffizienten $\tilde{s}$ verwendet (7). Dessen niedriger Wert von 0,81 überrascht nicht, wenn man feststellt, daß nach zweidimensionaler Entwicklung auf der Dünnschichtplatte Corticosteron und Prednisolon eben angetrennt sind, während im Flüssigchromatographen beide Stoffe eine Zeitdifferenz von etwa 10 Minuten bei einer Peakhalbwertsbreite von 18 bzw. 60 Sekunden trennt. Dies äußert sịch auch in der Steigung der Geraden, wenn man auf einer Achse des Koordinatensystems die Werte mit Zwischentrennung, auf der anderen Achse die direkt gemessenen Zahlen aufträgt und durch die so gewonnenen Meßpunkte die Ausgleichsgerade legt (Abb. 5). Die Übereinstimmung mit Zahlenwerten, die nach der Proteinbindungsmethode (11) erhalten wurden, ist befriedigend (Tab. 4).

\section{Präzision}

Mehrfachanalysen reiner organischer Lösungen und von Extrakten von Reinsubstanzen ergaben eine Standardabweichung von $\pm 6 \%$. Ein Humanserum wurde zunächst $30 \mathrm{mal}$ analysiert. Dabei wurde eine Cortisolkonzentration von $76 \pm 7 \mu \mathrm{g} / 1$ ermittelt. Bei Doppelbestimmungen innerhalb von weiteren 6 Arbeitstagen wurden Werte

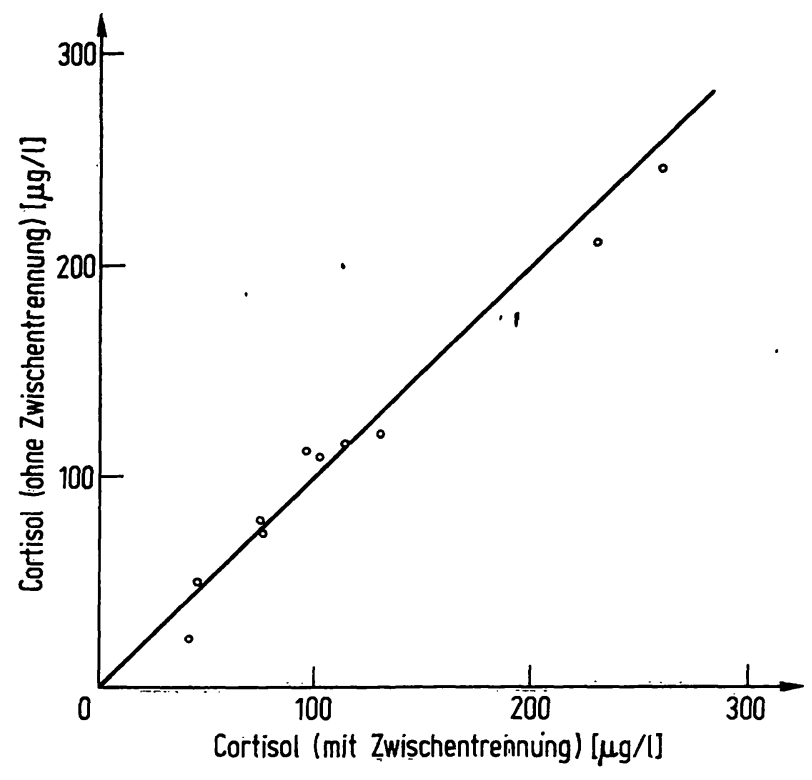

Abb. 5. Parallelanalysen von Humanseren ohne und mit dünnschichtchromatographischer Zwischentrennung.

Tab. 4. Vergleichsuntersuchungen von Humanseren mit der Flüssigchromatographie und nach der Proteinbindungsmethode

\begin{tabular}{cc}
\hline Flüssigchromatographie & $\begin{array}{c}\text { Proteinbindung } \\
\text { Cortisol }[\mu \mathrm{g} / \mathrm{i}]\end{array}$ \\
\hline 36 & 47 \\
43 & 80 \\
51 & 81 \\
70 & 72 \\
104 & 120 \\
115 & 11.8 \\
164 & 111 \\
179 & 181 \\
231 & 132 \\
266 & 200 \\
291 & 288 \\
297 & 340 \\
297 & 300 \\
211 & $300 *$ \\
\hline
\end{tabular}

* Prednisolon in großer Menge im Chromatogramm nachweisbar

zwischen 71 und $89 \mu \mathrm{g} / 1$ gemessen. Diese Zahlen liegen also innerhalb der dreifachen Standardabweichung der zuerst ermittelten Zahl.

\section{Empfindlichkeit}

Als eben noch erfaßbar soll hier ein Signal von der doppelten Höhe des Rauschpegels gelten. Das entspricht hier einer Signaländerung entsprechend einer Extinktion von 0,006. Da die Peakbreite der eluiẹten Zonen nur in sehr engen Grenzen und nicht mengenabhängig variierte, wurde die Erfassungsgrenze aus der Peakhöhe eines gut meßbaren Peaks, aus seiner Substanzmenge und dem entsprechenden Rauschsignal berechnet. Bei einer Probenmenge von $20 \mu \mathrm{l}$ beträgt sie für Corticosteron $2 \mathrm{ng}$, für Cortisol $4 \mathrm{ng}$. Da die Proben, um in das Gerät eingeführt werden zu können, auf $40-50 \mu \mathrm{l}$ aufgefüllt werden müssen, entspricht das einer Menge von $5 \mathrm{ng}$ bzw. $10 \mathrm{ng}$ 
Hormon. Der statistische Fehler der Messung beträgt hier $100 \%$, geht aber schon bei einem Signal vom funffachen Rauschpegel auf $20 \%$ zurück. Es ist ohne Modifikation der Extraktionsmethode möglich, eine größere Menge Serum zur Analyse einzusetzen, so daß auch Hormonspiegel im unteren Bereich gemessen werden können. Bei einem Probeneinsatz von $1 \mathrm{ml}$ Humanserum entspricht die oben mitgeteilte Erfassungsgrenze jedoch bereits einem Wert von $10 \mu \mathrm{g} / 1$. Bei Ratten reichte die Empfindlichkeit der Methode bei einer Probenmenge von 0,5 ml in jedem Fall aus. Es erscheint möglich, durch noch kürzere, höchstwirksame Säulen und durch eine weitere Verminderung des Rauschpegels eine weitere Senkung der Erfassungsgrenze bis um den Faktor $10 \mathrm{zu}$ erreichen.

Als großer Vorteil der Methode kann die Schnelligkeit angefuihrt werden, mit der die Analysenergebnisse vorliegen, da keine Äquilibrierungszeiten notwendig sind, außerdem ihr großer dynamischer Bereich, der es gestattet, auch große Konzentrationsunterschiede in einem Chro: matogramm zu erfassen. Mit jedem Chromatogramm wird gleichzeitig eine qualitative Information über die Steroide erhalten, die außer den gesuchten Hormonen noch in der Probe vorhanden sind. Das aufwendigste Gerät, der Flüssigkeitschromatograph, läßt sich ohne Umbau auch für andere Bestimmungen einsetzen. Es ist zu erwarten, daß in Kürze auch andere Parameter mit dieser Methode bestimmt werden können. Ein Schritt in diese Richtung ist die Bestimmung biogener Amine als Acetylderivate aus reiner Lösung, die auf biologisches Material übertragen wird (12).

\section{Danksagung}

Herrn Dr. R. Berg, Institut für Klinische Biochemie der Universität Bonn, danken wir für Plasmaproben, dic nach der Proteinbindungsmethode voranalysiert waren, Herrn Dr. H. Schlebusch, Univ.-Frauenklinik Bonn, für die Überlassung von Serumpools. Die Arbcit wurde aus Mitteln des Bundesministeriums für Jugend, Familic und Gesundheit, Gcsch.-Nr. L II 5-48200, gefördert.

\section{Literatur}

1. Bean, W. B., Hodges, R. E. \& Daum, K. (1955), J. Clin. Invest. 34, 1073-1084.

2. Henry, R. A., Schmit, J. A. \& Dieckman, J. F., (1971) J. Chromatogr. Sci. 9, 513-520.

3. Meijers, C. A. M., Hulsman, J. A. R. J., \& Huber, J. F. K., (1972) Z. Anal. Chem. 261, 347-353.

4. Siggia, S. \& Dishman, R. A., (1970) Anal. Chem. 42, 12231229.

5. Touchstong, J. C. \& Wortman, W., (1973) J. Chromatogr. $76,244-247$.

6. Hesse, C. \& Hövermann, W., (1973) Chromatographia 6, 345-348.
7. Pirke, K. M \& Stamm, D., (1972) diese Z. 10, 243-253.

8. Kaiser, R., (1965) Chromatographie in der Gasphase, Bd. IV, quant itative Auswertung, S. 209-213, Bibliographisches Institut, Mannheim (B.I.-Hochschultaschenbücher 92/92a).

9. Huber, J. F. K., (1968) Influence of the separation process on the detection limit, Symposium on the limits of detection, Enschede.

10. Pietrzik, K., Hesse, C., Schulze zur Wiesch, E. \& Hötzel, D., Nahrung, im Druck.

11. Murphy, B. E. P., (1967) J. Clin. Endocrinol. 27, 973-989.

12. Roeder, E., Merzhäuser, J. \& Hesse, C., (1973) Klin. Wochenschr. 51, 883-884.
Dr. Christof Hesse
53 Bonn
Endenicher Allee 11-13 